\title{
Time Domain Passivity Control-based Telepresence with Time Delay
}

\author{
Jordi Artigas, Jordi Vilanova, Carsten Preusche and Gerd Hirzinger \\ DLR - German Aerospace Center \\ Institute of Robotics and Mechatronics \\ Munich, Germany \\ Email: jordi.artigas@dlr.de
}

\begin{abstract}
This paper analyses the Time Domain Passivity Control approach in the time-delayed telepresence context, and proposes a method which provides stable operation. The passivity controller for the two-port network which is created by the bilateral control and communication elements in [1] is shown to be not valid if a time delay is introduced in the communication channel. Classical stability analysis for the delayed system is presented and used as argument and benchmark for the proposed solution. Simulations and experimental results are discussed and compared with classical stability analysis.
\end{abstract}

\section{INTRODUCTION}

Telepresence is an extension of the telerobotics concept in which a human operator is coupled with as much sensory information as possible to a remote environment through a teleoperator, in order to produce an intuitive and realistic interaction with that environment. The range of senses can encompass vision, tactility, aural, and even olfactory senses. Our interest is focused on the haptic channel, which is of crucial importance in providing a realistic feeling, and therefore in performing a tele-present task. The haptic channel is a challenging topic due to its closed loop characteristics which includes the Human Operator (HO) in its force feedback path. Transparency, meaning in its ideal form that the $\mathrm{HO}$ is not able to distinguish local presence from telepresence, always relies on stability. Publications [2], [3] and [4] discuss the stability of the system and show how it compromises transparency when design constrains are established. This has become a classical trade-off which gets a special tedious shape when time delay is involved in the communication channel. A system which behaves stably in a delay-free scenario can easily become unstable in the delayed one. Often the solution to preserve stability involves a strengthening of the design constraints, leading to conservatism.

One of the most remarkable approaches in dealing with time-delayed bilateral control bases its concept on passivity criteria [5], [6], [1], [7], [8]. Passivity is a sufficient condition for stability and provides a mathematical tool which allows global stability conclusions to be drawn from individual block consideration. Therefore it can be used as a design rule for time-delayed telepresence control since the main problem with such systems is that the communication channel becomes a non-passive element. One of the most outstanding works of (frequency domain) passivity-based approaches is [5], where the system is modelled by means of scattering parameters and transmission lines equations are used to design the bilateral controller. Likewise distinctive is its extension to the Wave Variables concept [6], which provides design rules and a solid method for time-delayed telepresence. The major problem with using passivity in the design process is that often such systems are over-conservative. In many cases performance can be poor if a fixed damping value is used to guarantee passivity under all operating conditions [1].

The method proposed in this paper is based on the Time Domain Passivity Control (TDP) approach presented by B. Hannaford and J.H Ryu in [9], initially thought for haptic interfaces interacting with virtual environments. This approach provides a mechanism to analyse passivity in real-time and bases its control on the real-time observability of the system energy. Thereby passivity is no longer a design constraint which fixes controller rules (and thus compromising transparency), but rather an on the fly observation and correction of passivity of the system. The approach presented in this article aims at the use of this mechanism to make the delayed communication element passive on the time domain (as opposed to scattering methods where the passivation takes place at the design process and in the frequency domain). It has already been shown how delayed communication channels may exhibit an active nature [5], [6]. The TDP approach has already been applied to telepresence [1], [10]; however the time delayed scenario impose constraints which make these approaches unfeasible. This delayed scenario has not yet been considered and is the focus of this paper.

The outline of this paper is organised as follows. Section II reviews the TDP approach with a focus on the context presented in this article. A classical stability analysis of timedelayed systems is given in Section III in order to show the problem induced by the active nature of delayed communications and to give some hints for the later design. Section IV describes the telepresence scheme in terms of electrical circuits and network elements in order to examine and test the passivity of the communication channel. Section V presents the proposed solution and issues involved around it. Finally, experiments are conducted in Section VI followed by some conclusions in Section VII. 


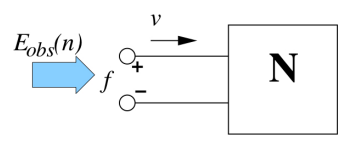

Fig. 1. Energy flow of a one-port network

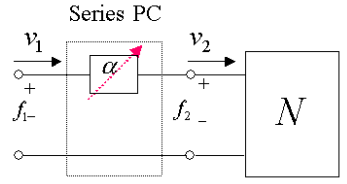

Series or velocity conserving

Impedance causality

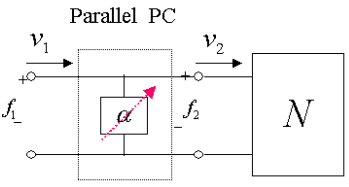

parallel or force conserving

Admittance causality
Fig. 2. The causality of the one-port network determines velocity or force conservation by the PC

\section{Review of Time Domain Passivity}

This section reviews the basic concepts of the TDP control. Loosely speaking, a system is said to be passive if the energy entering the system is greater than the outgoing energy. Using the sign convention for force and velocity indicated in Fig. 1, a one-port network is defined to be passive if and only if,

$$
\int_{0}^{t} f(\tau) v(\tau) d \tau+E(0) \geq 0, \quad \forall t
$$

where $v$ and $f$ are the port variables denoting velocity and force respectively, and $E(0)$ is the energy stored initially in the network element at $t=0$.

Hannaford and Ryu [9] introduced the concept of Passivity Observer (PO). In discrete systems the energy flow can be computed in real-time. For a one-port network the PO in the discrete domain is defined as

$$
E_{\text {obs }}(n)=\Delta T \sum_{k=0}^{n} f(k) v(k),
$$

where $E_{\text {obs }}(n)$ is the observed energy and $\Delta T$ is the sample time of the system. If $E_{o b s}$ is positive, the element will be passive. If $E_{o b s}$ is negative the element will be active. In order to keep the system passive, the amount of extra energy has to be dissipated. This is done by an additional element, the socalled Passivity Controller (PC): a damping element which varies as a function of the observed energy. Fig. 2 shows two possible configurations of PCs, depending on the causality of the network element.

In the telepresence context presented in this article, i.e. bilateral control of the haptic channel, the impedance causality is used, where position or velocity is read from the haptic device/slave robot, and the force is computed by the bilateral control blocks. In this case (series configuration) the variable damping factor of the PC $\alpha(n)$ is calculated as follows,

$$
\alpha(n)=\left\{\begin{array}{ll}
-\frac{E_{o b s v}(n)}{\Delta T v(n)^{2}} & \text { if } E_{\text {obsv }}(n)<0 \\
0 & \text { if } E_{\text {obsv }}(n) \geq 0
\end{array},\right.
$$

where $v(n)$ is the current velocity. For the parallel PC case, the damping parameter $\alpha$ is calculated similarly based on the current force. The reader is referred to [9] and [11] for the complete formulation of the TDP approach.

\section{ANALYSIS OF TIME-DELAYED HAPTIC CHANNEL}

Fig. 3 shows the telepresence scheme under study, a two channel symmetrical position-position system. The concepts presented in this paper are all based on these causalities; however, they are easily applicable to any other two-channel configuration. We begin by examining the open-loop transfer function in the Laplace domain $\left(G_{o l}\right)$. The system is created by master/slave devices, controllers, communication channel, and a free-space environment, for the non-delayed case:

$$
\begin{aligned}
G_{o l}= & \frac{v_{s}}{F_{h}}=\frac{N u m}{D e n} \\
N u m= & -\left(B^{2} s^{2}+2 K B s+K^{2}\right) \\
D e n= & m^{2} s^{4}+2 m(b+B) s^{3}+\left(2 m K+(b+B)^{2}\right) s^{2}+ \\
& 2(b+B) K s+K^{2} .
\end{aligned}
$$

Where master and slave are modelled with identical masses and viscosities ( $m$ and $b$ ) and $K$ and $B$ are the PD parameters. The extension to the delayed case is given by

$$
G_{o l_{d}}=G_{o l} e^{-s 2 T},
$$

where $T$ is the time delay in the communication channel, and is doubled due to the forward and feedback paths. The main concern targeted in this analysis tries to find out the values of the PD controllers parameters, $K$ and $B$, for which the system is stable for any possible delay $T$. Based on Hurwitz's stability criterion, the poles of the characteristic polynomial will determine the stability of the system. If all of them fall on the left half plane (LHP) of the root loci diagram, the system is stable. The characteristic polynomial of the system represented in Fig. 3 is given by

$$
1+G_{o l} e^{-s 2 T}=0,
$$

which takes the form of a second order transcendental equation in the variable $w$ but not on the delay variable, $T$. The roots can be computed by fixing $s=j w$, i.e. the imaginary axis crossing points or critical frequencies. Equating real and imaginary part to zero, the two critical pairs of frequencies

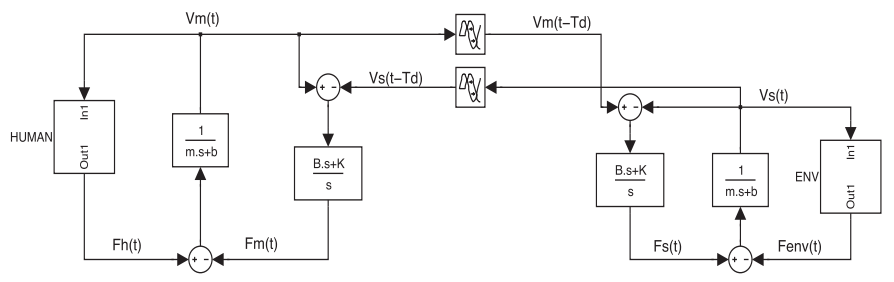

Fig. 3. Symmetric position/position PD control scheme with time delay 


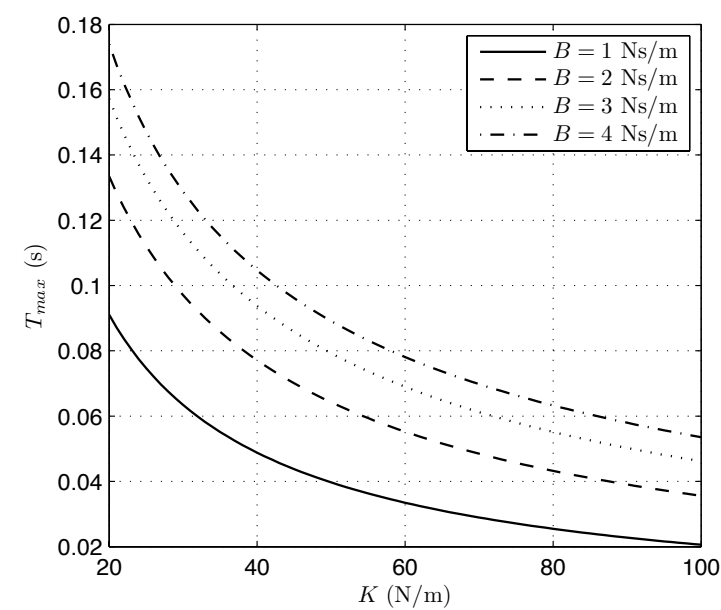

Fig. 4. Maximum allowed time delay as a function of the PD controller parameters. Stability regions are therefore below the curves. Master and slave parameters used are $m=0.1315 \mathrm{~kg}$ and $b=0.15 \mathrm{Ns} / \mathrm{m}$.

as a function of $T$ can be found, and thus two delays are encountered:

$$
T_{1,2}=\frac{\operatorname{ArcCos}\left[\frac{W(K, B, m) \pm \sqrt{X(K, B, m)}}{N(K, B, m)}\right]}{U(K, B, m, b)},
$$

where the terms $U(K, B, m, b), W(K, B, m), X(K, B, m)$ and $N(K, B, m)$ are used to simplify the above equation. These analytical expressions (eq. 7) are of eminent interest, namely they let us explore what is the maximum time delay allowed for the given system, or to investigate how the system evolves when a parameter is varied. Fig. 4 shows these stability regions drawn by eq. (7) as a function of the PD controllers parameters.

This analysis gives some design hints which can indeed ensure stable operation for the time-delayed system. However the system is only feasible for a reduced set of external conditions (i.e. fixed and known time delay and free space environment). An operating point on the stability boundary is very susceptible to go unstable if any of the external conditions vary (a stiff contact in the environment, an increase of the communication delay). To properly cope with the system's stability covering all possible range of external conditions, robust stability criterions should be applied, which in turn, often result in over-conservative solutions (and thus lowering transparency by fixing very high damping values $(B)$, and low position parameter $(K)$ ).

The next sections present a solution based on the one-port passivity controller, which allow the designer to set up an operating point close to the boundary stability region while obtaining a stable performance when external conditions (time delay) are changed.

\section{Circuit Representation And Passivity Test}

This section puts forward some considerations which hold and justify the lines presented in the coming sections. In the previous section, stability has been explored from a classical control theory point of view. Although passivity is traditionally a more conservative approach than stability criterions (i.e. a system can be non-passive though stable) it provides a more suitable tool to draw conclusions about stability in the telepresence framework [5], [6]. Specifically it is interesting to underline the passivity property of connected network elements:

Theorem 1: If each of the individual network elements of a system are passive, the global system will be passive. Moreover, passivity is a sufficient condition for stability.

Fig. 5 shows the network representation of the system sketched in Fig. 3. Using the mechanical-electrical analogy [5], [9] our system can also be illustrated as in Fig. 6.

Note how the communication block is represented by means of two dependent current power sources.

\section{A. Two-Port PO}

The analysis proposed here is based on an online monitoring of the energy flow of the constituent network elements. The tool used for this monitoring is the two-port Passivity Observer element, proposed by [1], which computes the energy flow in the time domain as

$$
E_{o b s}(n)=\Delta T \sum_{k=0}^{n}\left[f_{1}(k) v_{1}(k)+f_{2}(k) v_{2}(k)\right],
$$

where $f_{1}, v_{1}, f_{2}$ and $v_{2}$ are the conjugate pairs on both ports respectively.

\section{B. Activity of the Communication Channel}

The Human Operator (HO) is the first source of energy. If the communication was not delayed, this energy would be captured by the haptic device, transmitted through the communication channel and dissipated on the slave side (assumed that master and slave devices and bilateral controllers are passively designed). However, as already mentioned, the delayed communication may break with the passivity formalism. Fig. 7 shows the energy flow for a simulated communication channel with a delay of $0.1 \mathrm{~s}$. Note how energy is first accumulated and soon releases. As a result, the communication channel becomes an active element. If the bilateral control is included in the two-port network element the block is more dissipative.

\section{Consideration and Assumptions}

The following passivity related considerations and assumptions can be stated for the component elements:

- HO: By definition this is an active element, since it is the first source of energy. It is commonly assumed that this

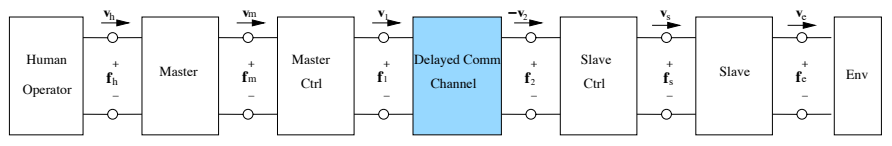

Fig. 5. Network representation of the telepresence system 


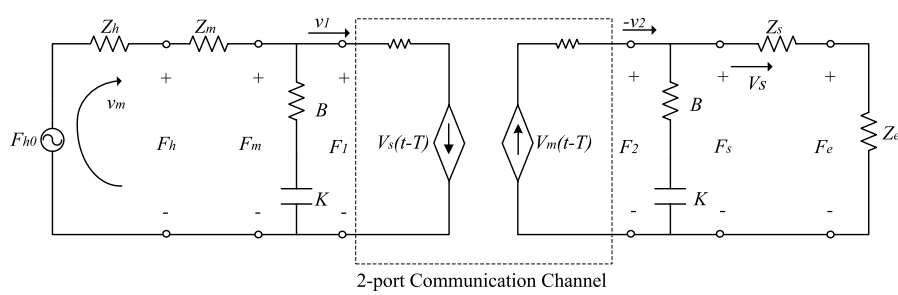

Fig. 6. Circuit representation of the telepresence system

activity will not contribute to instability, since the $\mathrm{HO}$ will behave in a stable manner.

- Master and slave devices are designed to be passive. It is assumed that their mechanical nature can not make them active due to their inherent dissipative elements.

- Both, master and slave controllers may be active since they often induce a phase lag between input and output and they are responsible for moving master and slave devices [10]. However, since our focus is placed on the passivity of the communication channel, a passive design of the controllers is considered [12].

- The environment may be active. Only passive environments are considered.

- Communication Channel: if a transmission delay is involved, the two-port network may become active [5].

\section{Inconsistency of of Two-Port PO/PC}

In [1] the channel and the bilateral control blocks are considered as parts of the same network element. In contrast, here the channel is isolated from the rest of the system and its passivity is solely investigated since this is the concern in this paper. The test shows empirically the activity of the communication element. The approach presented in [1] assumes there is no transmission delay between port one and two. Thereby eq. 8 is consistent, and the development of a two-port based Passivity Controller which acts simultaneously on both ports is feasible. Nonetheless, the nature of delayed communication channels does not allow this approach; mainly for two reasons:

- The energy of a two-port network can not be properly observed because the conjugate pair information of both ports can not be simultaneously present at one specific location, and therefore it is not consistent with eq.(8).

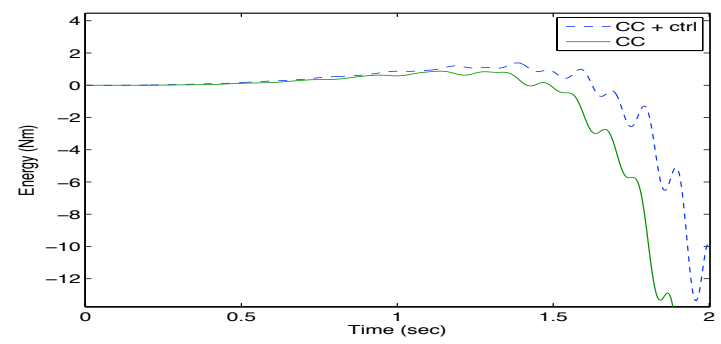

Fig. 7. Energy flows in the communication channel (CC) and in the communication + bilateral controller $(\mathrm{CC}+\mathrm{ctrl})$
- The two variable damping factors of the Passivity Controller of port 1 and port 2, again cannot be simultaneously applied because of the temporal distance.

These two arguments motivate the examination of other TDP-based solutions which target the passivation of delayed communication channels.

\section{Proposed Solution}

The main idea is illustrated in Fig. 8, where the one-port network definition of the passivity controller is used in the telepresence context. Since the communication channel is the main active block, the PO/PC elements should be able to observe and release the extra energy resulting from the delayed communications. As it can be seen, two different network topologies are combined to create two one-port passivity controllers representing one bilateral controller. The proposed method is described through the following subsections:

\section{A. Forward Passivity Controller}

The network lumps the following elements: master PD control, communication channel, slave PD control, slave robot and environment. The forward passivity controller (PCF) ensures that the overall system is passive. However, if the energy flow generated by the activity of the communication channel goes from left to right, the slave can easily become unstable because no one is ensuring a passivity correction at the slave port. This is a limitation, and proves that the PCF as a standalone mechanism is not sufficient to overcome the problems induced by the delayed operation.

\section{B. Backward Passivity Controller}

In a similar manner, the backward network can be defined covering the elements located on the master side together with the communication channel. The backward passivity controller (PCB) has the analogous problem as the one described for the PCF, being therefore not sufficient as a standalone controller. Moreover, a sharper look at the topology reveals an added problem: as already mentioned the HO is a source of energy by definition. This energy is captured by the backward network PO indicating active behavior. This means that, given the nondelayed scenario, the PCB is activated when the HO performs a movement. This may add some undesired extra damping.

\section{Bilateral Passivity Controller}

The logical reasoning after examining both, PCF and PCB, is to combine both. This topology solves the problems observed in the two previous configurations, i.e. assures passivity

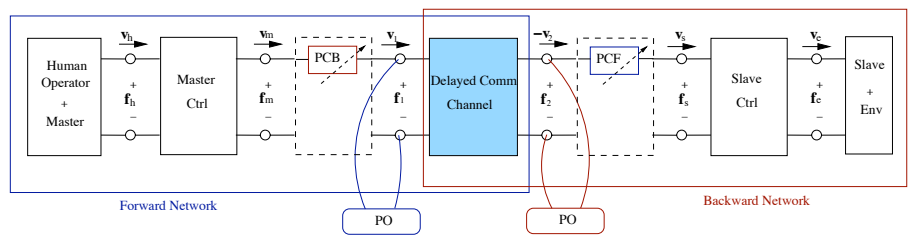

Fig. 8. Two faced one-port networks create the Bilateral Passivity Controller (PCBI) 


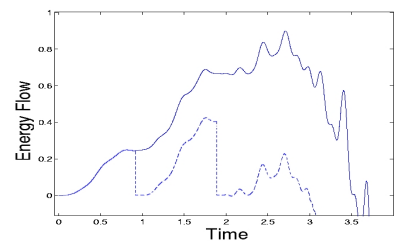

Fig. 9. Solid: Accumulated energy slows PC's reaction. Dashed: Resetting effects over acummulated energy; the system reacts faster.

in the time domain of the communication element for any direction of the energy flow.

\section{Passivity Observer and Energy Resetting}

The PO introduced in [11] is used, where the constant force or velocity assumption is removed. One of the main problems in using the TDP approach for time-delayed systems is the fact that because of the nature of such systems, some energy is accumulated in the channel. This can make the system vulnerable to instability because the reaction time which the energy needs to get from the accumulated value to the negative value is often too large. That makes the PC too slow to react against activity. Fig. 9 shows a plot of the observed energy flow at one of the ports of a time-delayed system. It should be noted that up to one point the upwards tendency changes, indicating that the flow inside the network is changing. However, the PC does not react until $t=3.5 s$, the moment in which the energy drops lower than zero. An energy resetting element is proposed driven by the following two conditions:

$$
\begin{aligned}
& f<f_{t h} \\
& t>t_{t h} .
\end{aligned}
$$

Where $f$ denotes the force being used for the energy monitoring, $t$ is time, and $f_{t h}$ and $t_{t h}$ are force and time threshold. The effects of this mechanism are shown in Fig. 9, where the PC can react at $t=3 \mathrm{~s}$. Subsection V-E gives some hints on how to choose the threshold values.

\section{E. PCBI design methodology}

The process can be synthesised within the following steps:

1) PD controllers design: The PD controllers should ensure stability for the free-space environment and non-delayed communication channel. The analysis presented in Section III gives a reference on how to choose specific values of $K$ and $B$. As a suggested rule, a working point close the stability regions shown in Fig. 4 will assure stability for the nondelayed context and will make the PCBI work for the delayed one.

2) Resetting thresholds: The values of the thresholds, $t_{t h}$ and $f_{t h}$, are chosen by observing the characteristics of the delayed channel. As a design hint, $f_{t h}$ should be slightly higher than the damping associated due to the non-delayed free environment and smaller than the lightest possible contact force. The value of $t_{t h}$ requires likewise an empirical approach. The time of the accumulated energy drop due to
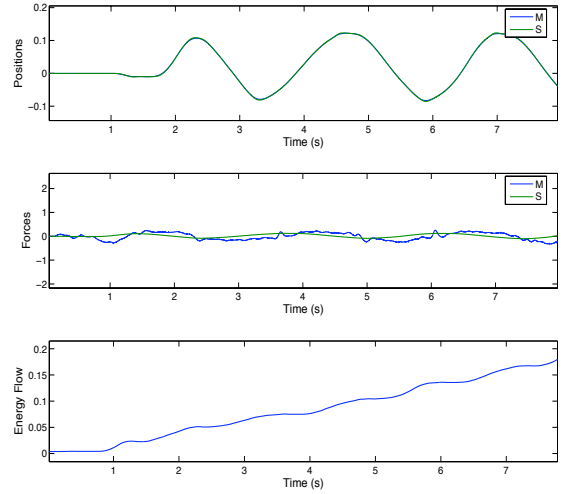

Fig. 10. "ideal" non-delayed free-space environment plots.
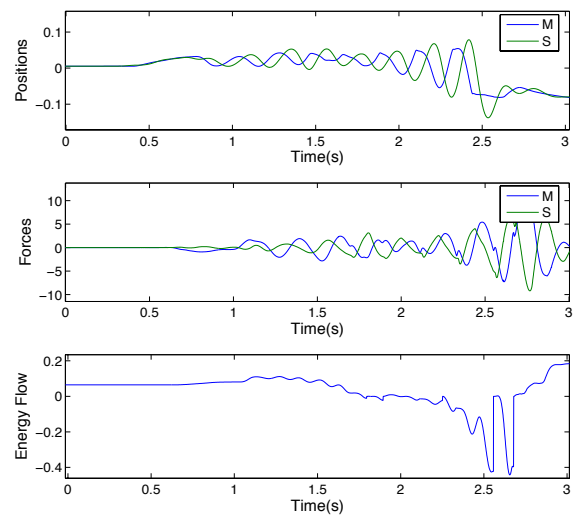

Fig. 11. Effects of the the time delay introduced in the channel. Active system, therefore unstable.

the time delay is a reference which can be used for setting $t_{t h}$. See Fig. 9.

\section{EXPERIMENTAL EXAMPLE}

The system described in Fig. 8 has been implemented in order to experimentally validate it. A 6 DoF $(3$ active +3 passive) PHANToM haptic device driven by a RT-Linux 3.3 at a sampling rate of $1 \mathrm{kHz}$ was used as master device. The communication channel is a UDP connection where a circular buffer was implemented in order to simulate a wide variety of time delays. The slave side was implemented within a Linux in which a 3 DoF robot with similar characteristics to the PHANToM has been simulated. The environment is likewise simulated providing free space situations and different wall stiffness. The parameters of both PD controllers are chosen by using the criterion described in Section V-E: $B=4 \mathrm{Ns} / \mathrm{m}$ and $K=55 \mathrm{~N} / \mathrm{m}$, which are located in the unstable vicinity of the stability boundary.

For each of the experiments, position, force and energy plots are given. We first show how the system behaves on the nondelayed scenario and a free-space movement (Fig. 10). It can be seen how the system is passive by looking at the energy 

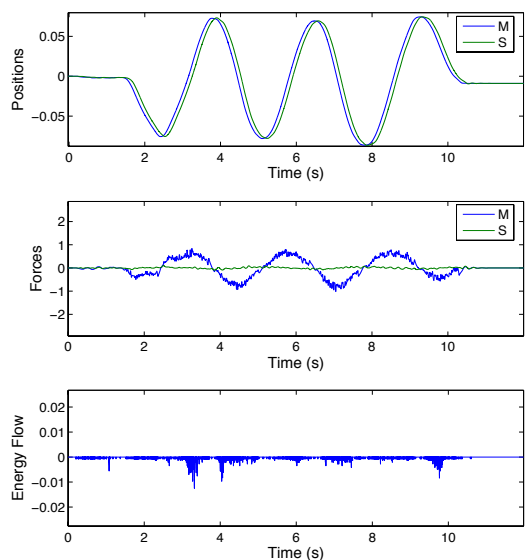

Fig. 12. Free-space environment with PCBI and a constant delay of $100 \mathrm{~ms}$.

flow plot, which is referred to the port corresponding to the backward network. A constant delay of $100 \mathrm{~ms}$ was introduced in the communication channel. The unstable behavior caused by a simple movement of the PHANToM device on the freespace environment is depicted in Fig. 11. Note the active behavior shown in the energy flow plot. The stabilising effects of the PCBI can be seen in Figs. 12 and 13. The system shows stable operation on the free-space as well as on a wall contact situation. However, as it can be seen in Fig. 13, the slave incurs a high frequency oscillation in position and force due to the activation/deactivation nature of the of the passivity controllers.

\section{CONCLUSIONS AND FUture WORK}

The paper presents an approach which circumvents the frequency passivity-based methods. By treating passivity in the time domain the telepresence system may be mainly aimed at transparency instead of at the classical trade-off which compromises transparency with stability. A controller which was tuned to be stable for the non-delayed scenario has been shown to remain stable in the delayed case by applying the PCBI. Moreover, the adaptive nature of the approach makes the system valid for a wide range of communication channels. The problem observed in the wall contact experiment (Fig. 13) suggests a solution based on the reference energy approach [13], where the fixed zero energy threshold is replaced with a time varying reference energy behavior to avoid exciting high frequencies of the system. Future work will concentrate on the feasibility of the energy reference approach within the PCBI framework, and will experiment with variable delay channels such as the internet, or radio links encountered on space telepresence. Currently, efforts are on to apply the approach on an internet-based telepresence system which will couple two 6DoF PHANToM, as well as on a ISS ${ }^{1}$ space telepresence experiment [14].

\footnotetext{
${ }^{1}$ International Space Station
}
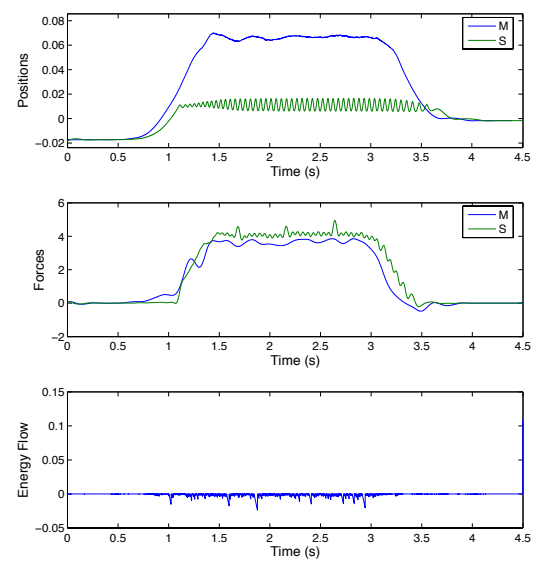

Fig. 13. Wall contact environment with PCBI and constant delay of $100 \mathrm{~ms}$.

\section{REFERENCES}

[1] J.-H. Ryu, D.-S. Kwon, and B. Hannaford, "Stable Teleoperation with Time Domain Passivity Control," in IEEE Intl. Conference on Robotics and Automation, ICRA, Washington, DC, USA, May 2002, pp. 3260-65.

[2] D. A. Lawrence, "Stability and Transparency in Bilateral Teleoperation," IEEE Transactions on Robotics and Automation, vol. 9, no. 5, IEEE Transactions on Robotics and Automation 1993.

[3] B. Hannaford, "A Design Framework for Teleoperators with Kinesthetic Feedback," IEEE Transactions on Robotics and Automation, vol. 5, no. 4, pp. 426-434, August 1989.

[4] Y. Yokokohji and T. Yoshikawa, "Bilateral Control of Master-Slave Manipulators for Ideal kinesthetic Coupling - Formulation and Experiment," IEEE Transactions on Robotics and Automation, vol. 10, no. 5, pp. 605-620, October 1994.

[5] R. J. Anderson and M. W. Spong, "Bilateral Control of Teleoperators with Time Delay," IEEE Transactions on Automatic Control, vol. 34, no. 5, pp. 494-501, May 1989.

[6] G. Niemeyer, "Using wave variables in time delayed force reflecting teleoperation," Ph.D. dissertation, Massachussetts Institute of Technology, September 1996.

[7] S. Hirche and M. Buss, "Telepresence control in packet switched communication networks," in Proceedings of the IEEE International Conference on Control Applications, Taipei, Taiwan, 2004, pp. 236-241.

[8] J. Artigas-Esclusa, C. Preusche, and G. Hirzinger, "Wave Variablesbased Bilateral Control with a Time Delay Model for Space Robot Applications," in Robotik 2004, VDI-VDE, Ed., Germany, 2004.

[9] J.-H. Ryu, D.-S. Kwon, and B. Hannaford, "Stability Guaranteed Control: Time Domain Passivity Approach," in Proceedings of the 2002 IEEE/RSJ Intl. Conference on Intelligent Robots and Systems, Lausanne, Switzerland, October 2002, pp. 2115-21.

[10] J.-H. Ryu and J. Kim, "Stable and High Performance Teleoperation with Time Domain Passivity Control: Reference Energy Following Scheme," in Int. Conf. on Advanced Robotics 2005, Seattle, USA, 2005.

[11] J.-H. Ryu, D.-S. Kwon, and B. Hannaford, "Sampled- and ContinuousTime Passivity and Stability of Virtual Environments," IEEE Transactions on Robotics, vol. 20, no. 4, August 2004.

[12] C. Ott, A. Albu-Schaeffer, A. Kugi, S. Stramigioli, and G. Hirzinger, "A Passivity Based Cartesian Impedance Controller for Flexible Joint Robots Part I: Torque Feedback and Gravity Compensation," in Proc. IEEE International Conference on Robotics and Automation ICRA, New Orleans, USA, April 2004, pp. 2659-2665.

[13] J.-H. Ryu, B. Hannaford, C. Preusche, and G. Hirzinger, "Time Domain Passivity Control with Reference Energy Behavior,' in Proceedings of the 2003 IEEE/RSJ Intl. Conference on Intelligent Robots and Systems, Las Vegas, Nevada, USA, October 2003.

[14] C. Preusche, D. Reintsema, K. Landzettel, and G. Hirzinger, "Robotics Component Verification on ISS ROKVISS - Preliminary Results for Telepresence," 2006, unplublished. 